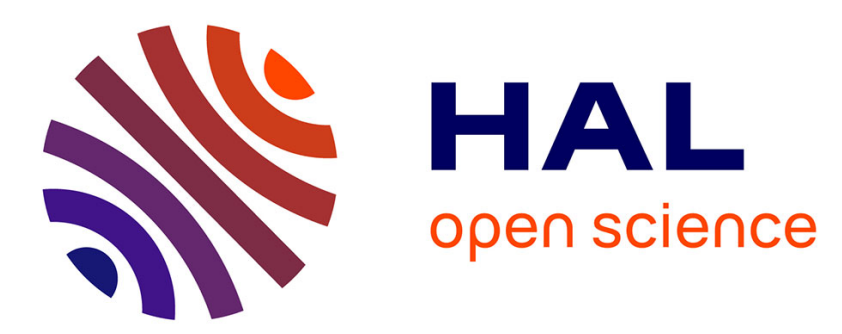

\title{
Evaporation of a sessile droplet: Inside the coffee stain
}

Guillaume Berteloot, Anna Hoang, Adrian Daerr, H. Pirouz Kavehpour, Francois Lequeux, Laurent Limat

\section{To cite this version:}

Guillaume Berteloot, Anna Hoang, Adrian Daerr, H. Pirouz Kavehpour, Francois Lequeux, et al.. Evaporation of a sessile droplet: Inside the coffee stain. Journal of Colloid and Interface Science, 2012, 370 (1), pp.155 - 161. 10.1016/j.jcis.2011.10.053 . hal-01576678

\section{HAL Id: hal-01576678 \\ https://hal.sorbonne-universite.fr/hal-01576678}

Submitted on 17 Nov 2019

HAL is a multi-disciplinary open access archive for the deposit and dissemination of scientific research documents, whether they are published or not. The documents may come from teaching and research institutions in France or abroad, or from public or private research centers.
L'archive ouverte pluridisciplinaire HAL, est destinée au dépôt et à la diffusion de documents scientifiques de niveau recherche, publiés ou non, émanant des établissements d'enseignement et de recherche français ou étrangers, des laboratoires publics ou privés. 


\title{
Evaporation of a sessile droplet : inside the coffee stain
}

\author{
Guillaume Berteloot $^{\mathrm{d}}$, Anna Hoang ${ }^{\mathrm{d}}$, Adrian Daerr ${ }^{\mathrm{e}}$, H. Pirouz \\ Kavehpourd ${ }^{\mathrm{d}}$,Francois Lequeux ${ }^{\mathrm{f}}$, Laurent Limat ${ }^{\mathrm{e}}$ \\ ${ }^{a}$ Complex Fluids and Interface Physics Laboratory,Department of Mechanical and \\ Aerospace Engineering, UCLA, Los Angeles, CA, 90095,USA \\ ${ }^{b}$ Laboratoire Matière et Systèmes Complexes, UMR 7057 CNRS \& UniversitéParis \\ Diderot, 10 rue Alice Domon et Léonie Duquet, 75013 Paris, France, EU \\ ${ }^{c}$ Laboratoire Physico-chimie des Polymères et Milieux Dispersés,UMR 7615 CNRS, \\ ESPCI 10 rue Vauquelin, 75005 Paris, France, EU
}

\begin{abstract}
We have investigated experimentally, for the first time at microscopic level, the growth of the deposit left around a drop of colloids drying on a solid surface ("coffee stain effect"). Direct observations show that there are several distinct phases of growth, the later ones exhibiting surprising pattern formations with spatial modulation of the deposit. In addition, fluorescence reveals that the initial growth phase is governed by a single length scale, increasing with time as $t^{\frac{2}{3}}$. We show that this exponent is a direct consequence of the divergence of evaporation near contact line evidenced by Deegan et al. We propose a simple ballistic model that allows us to calculate both this exponent and the prefactor, in agreement with yet available more complex descriptions. This model also opens the possibility to include effects neglected up to now.
\end{abstract}




\title{
Evaporation of a sessile droplet : inside the coffee stain
}

\author{
Guillaume Berteloot $^{\mathrm{d}}$, Anna Hoang ${ }^{\mathrm{d}}$, Adrian Daerr ${ }^{\mathrm{e}}$, H. Pirouz \\ Kavehpour $^{\mathrm{d}}$, Francois Lequeux ${ }^{\mathrm{f}}$, Laurent Limat $^{\mathrm{e}}$ \\ ${ }^{d}$ Complex Fluids and Interface Physics Laboratory,Department of Mechanical and \\ Aerospace Engineering, UCLA, Los Angeles, CA, 90095,USA \\ ${ }^{e}$ Laboratoire Matière et Systèmes Complexes, UMR 7057 CNRS \& UniversitéParis \\ Diderot, 10 rue Alice Domon et Léonie Duquet, 75013 Paris, France, EU \\ ${ }^{f}$ Laboratoire Physico-chimie des Polymères et Milieux Dispersés, UMR 7615 CNRS, \\ ESPCI 10 rue Vauquelin, 75005 Paris, France, EU
}

\section{Introduction}

Coating a solid surface with colloid layers of uniform thickness is a challenge of central importance in many industrial applications. A typical example is the control of optical properties of glasses which can be tuned at will with appropriate colloidal compounds $[1,2]$. Several methods can be imagined for such coatings on large scale surfaces : dip-coating [3], i.e. drying of a film entrained by a plate pulled out of a bath, or drying of a film left on a plate by a moving inclined blade [4], etc.

However, the interaction between deposition, evaporation and hydrodynamics is a complex matter. A previous study [5] showed that one can predict different regimes for dip-coating due to evaporation. As the presence of the contact line seems to play a crucial role in coating with an organized deposit [6], we need more experiments to get a better understanding of the mechanisms involved.

The simplest possible experiment is the drying of a droplet on a solid substrate. This phenomenon has been studied macroscopically [7, 8], and 
evaporation was found to be diverging at the contact line, providing an explanation for the famous "coffee stain" : as well-known, extremely high evaporation rate, which is modeled as a singularity using an electrostatic analogy [8], drives particles to the contact line, thus forming a ring all around the drop once the liquid has dried out.

To our knowledge, despite the great practical and fundamental interest of this problem, there is no available direct microscopic visualisation of the deposit while it is growing, in real time conditions. If we exclude the case of polymer deposition[9] and drying micelles [10], most of available works have been made a posteriori with macroscopic observations or measurements, and nobody looked at the details of the growth, i.e. on how the deposit builds itself microscopically. In this letter, we investigate these processes by combining microscopy with the use of fluorescent particles, providing to our knowledge a first direct measurement of a growing deposit. We show that the growth of the deposit involves several distinct phases, each one possessing its own dynamics. We show that in the first phase, both the deposit width and thickness increases with time, following the same power law with time : $x_{0}(t) \approx h_{0}(t) \approx t^{\frac{2}{3}}$. We recover this exponent and calculate the prefactor by building a simple model in which the deposit grows as if one was filling a wedge of constant contact angle, the particle being driven balisticaly by the evaporation. This law has been earlier proposed in $[11,12,13]$ with three different methods, but we improve strongly here that of Rio et al. [13] by first correcting mistake in its prefactor, and then by including in the model the fact that the evaporation singularity can also move with the deposition front. At longer time scales, the structure of the deposit becomes more complex, with 
presumably the formation of a "skin" floating at the free surface of the drop, while the front velocity strongly increases. Simultaneously, surprising pattern formation is observed on the deposit in the later stages of growth, that we attribute to a possible shear-banding or buckling of the deposit induced by the mechanical constraints exerted by the flow. This structuration of the deposit is followed at even longer time scales by the well known fracturation and delamination behaviors reported by other authors $[14,15]$.

In the present work, the possible influence of Marangoni flows hase been neglected, though some qualitative observations that we have made with fluorescent particles suggest the possible appearance of some recirculations in the liquid drop. Indeed, it has been shown $[16,17,18,19]$ that this kind of effect is expected to appear with possible modifications of the deposit structure when the thermal conductivity of the liquid differs from that of the substrate. Usually these effects are supposed to be negligible in water, as any contamination of its free surface should induce flows opposed to the previous ones [20]. We have left this question open for future studies.

In Section II, we briefly describe our experiments. General observations are described in Section III : different growth phases, deposit structure... A quantitative study of deposit growth, using fluorescence microscopy, is then presented in Section IV, and our simple model of the first stage of growth in Section V.

\section{Materials and methods}

To observe the growth of the colloidal deposit, we used a Nikon Eclipse TE 2000 inverted fluorescence microscope located at the CFIP laboratory in UCLA with a set of various objective lenses (ranging from $2 \times$ to $100 \times$ ) and a 


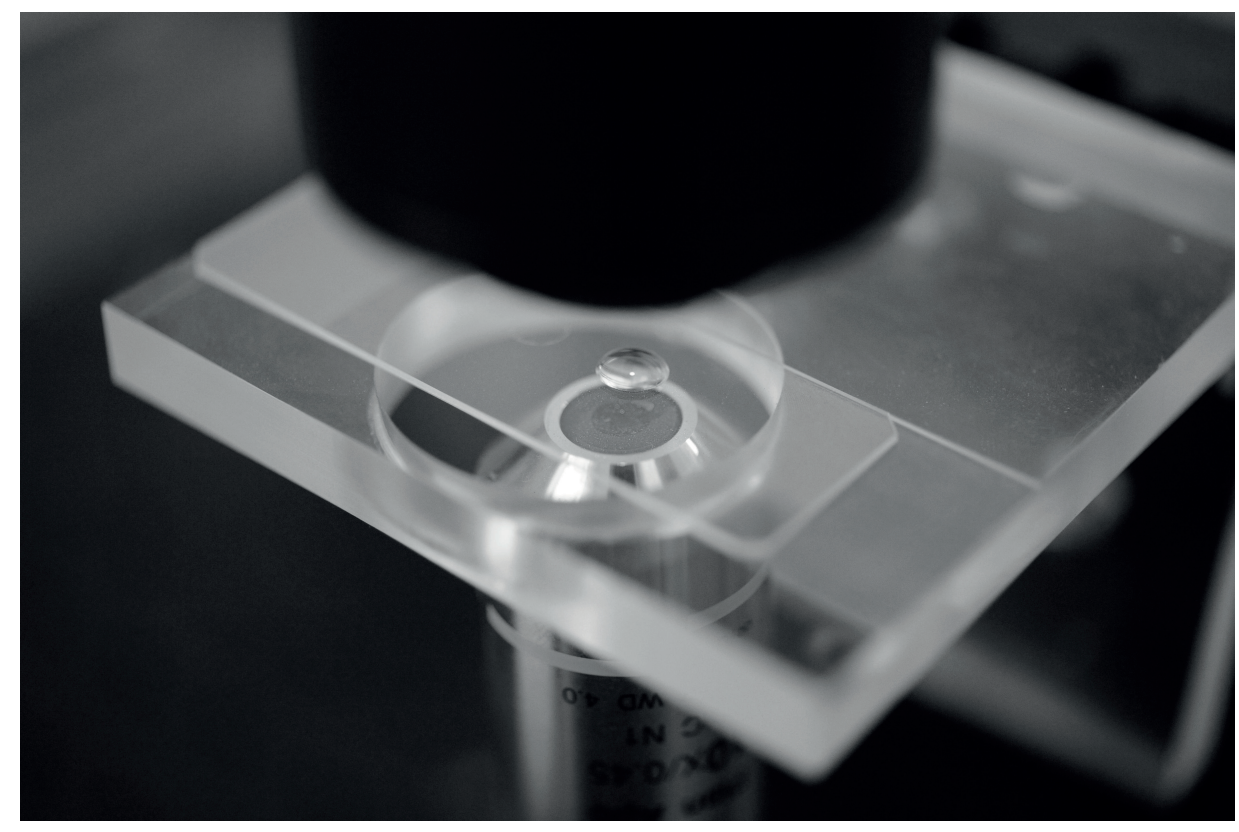

Figure 1: Experimental set-up : a droplet of $10 \mu \mathrm{L}$ of silica suspension is laid on a fresh microscope slide, and observed with an inverted microscope.

custom built microscope designed in the MSC laboratory in Université Paris Diderot, with a $20 \times$ objective lens. As a substrate, we used fresh untreated glass microscope slides. The colloidal solutions used - provided by Klebosolwere 50R50, 30R25 and 30R12 Klebosol silica slurries, which were diluted to reach our working concentration (in most cases, $2,5 \%$ in volume) using distilled water. We also used fluorescent silica particles of different radii. Volumes of $10 \mu \mathrm{L}$ of suspension were laid on the glass substrate, forming droplets of approximately $4 \mathrm{~mm}$ in diameter with a contact angle of $35^{\circ}$. The experiments were performed at room temperature, with a relative humidity of $20 \%$. Measurements were made using the Image Pro Plus software. 

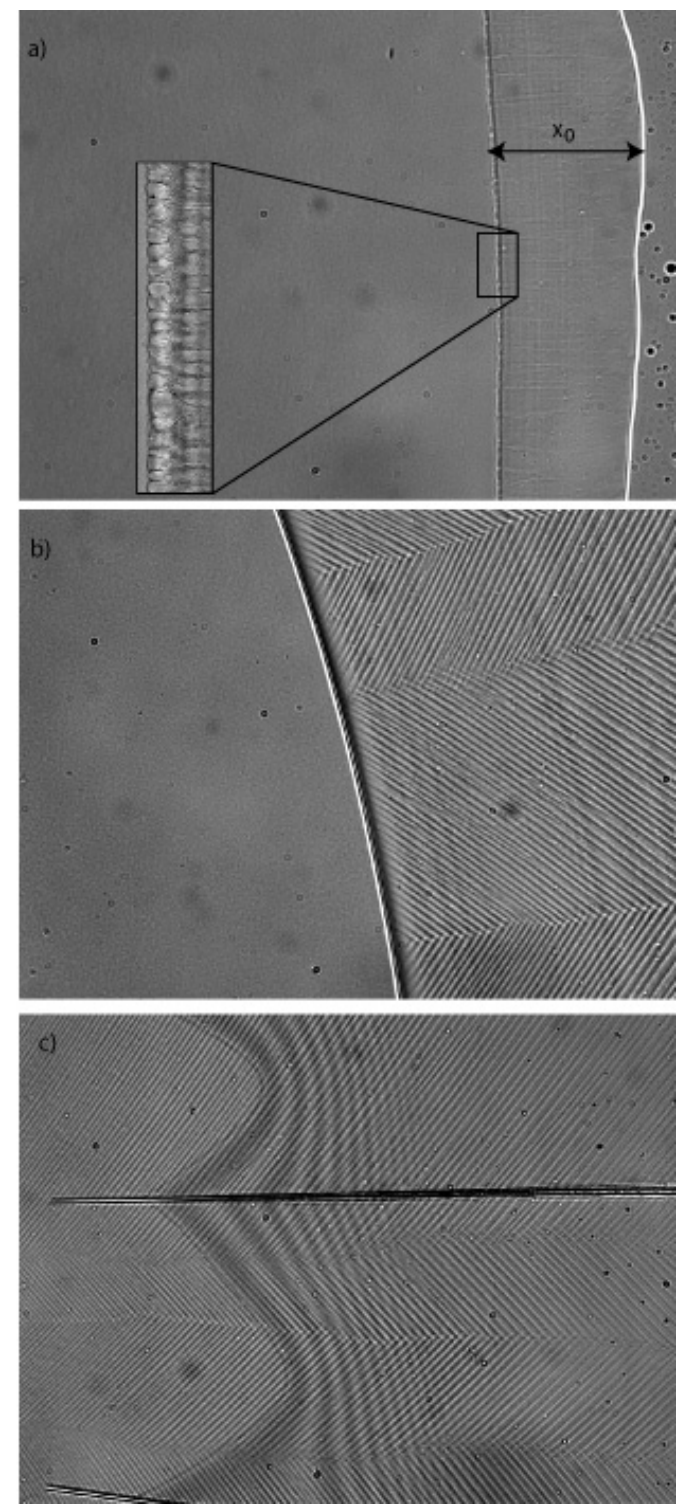

FiguRE 2: Drying of a solution of $50 \mathrm{~nm}$ particles observed with 40x objectives (each image is $350 \mu \mathrm{mx} 350 \mu \mathrm{m})$ : a) Initial growth of the deposit $(t=440 \mathrm{~s})$, the initial contact line can be seen in white (with unwetted glass on its right) while a deposition front appears and propagates to the center of the drop. Inset shows a $60 \times$ observation $(25 \mu \mathrm{m} \times 45 \mu \mathrm{m})$ of this deposition front. b) Formation of a stripe pattern, between the deposition front and the contact line (not visible on the picture) with stripes oriented at $45^{\circ}$ of the contact line $(t=5230 \mathrm{~s}) \mathrm{c})$ Cracks and delamination (visible from interference fringes) $(t=5497 \mathrm{~s})$. 


\section{Observations}

A typical experiment consists of a droplet of $50 \mathrm{~nm}$ particles, concentrated at $2,5 \%$, laid on a glass substrate, and observed using $20 \times$ magnification. The result is shown on 2-a) : we were able to detect the initial contact line, which remains always pinned in this work, and the growth of the colloidal deposit. In a time of the order of $10^{3}$ seconds, this deposit grows by a few hundreds of microns, the front exhibiting a nearly regular motion, altered by several retractions of about $10 \mu \mathrm{m}$. The landscape left behind the front is irregular, as observed by Kajiya et al[21] in the case of polymer sessile droplet. A closer observation of the deposition front, as shown in the inset of this picture, reveals the irregularity of this separation line, exhibiting a "fjordlike" shape. This shape is reminiscent of the one observed for solidification fronts [22], though a direct analogy is not so obvious. The deposition front also exhibits a few events of backward motion, reflecting a diminution in the spatial extent of the deposit, leaving marks parallel to the deposition front on the deposit, as visible on 2-a) and 2-b). This suggests that the deposit could be under compression, at least in the initial growth phase, compression that could be linked to the radial hydrodynamical flow induced by evaporation.

After a growth on order of one millimeter, stripes appear on the surface of the deposit, as shown on 2-b). These stripes get thinner and thinner and are oriented with an angle of $45^{\circ}$ to the contact line. The patterns have been observed using Atomic Force Microscopy (AFM), and the result using $50 \mathrm{~nm}$ particles is shown on 3. The surface of the deposit exhibits a height modulation, crests appearing between valleys. One can think about different explanations for this surface modulation : 
- The flow driving particles to the contact line puts the deposit under compression, and shear-banding can appear as for a metal under compression[23].

- As this deposit is still drying, thus losing volume, internal stress can appear that could lead to buckling.

- As we shall see later, in the late stage of its growth, the deposit might involve, close to the deposition front, a thin skin of colloidal particles floating on the liquid. This liquid will itself presumably recirculate below this skin, after depositing new particles at the front. The shear caused by this recirculation can put the skin under compression with a possible buckling, of typical "wavelength" close to the skin thickness. Stripes were always observed using $50 \mathrm{~nm}$ particles, only above $1,25 \%$ concentration for $25 \mathrm{~nm}$ particles, and never for $12 \mathrm{~nm}$ particles.

Once the deposition front has receded from a given area of the deposit, the liquid trapped inside it continues to evaporate, straining the material. As a result, cracks appear in the deposit, releasing the stress induced by loss of volume[24, 25, 26, 14]. Those cracks are visible on 2-c), and do not appear to have a correlation with the surface pattern. Of course, if the deposit is thin enough, strain will also be released through delamination, in which the deposit leaves the substrate and curves itself : this is plainly visible using the naked eye, but can also be seen on 2-c). As a matter of fact, interference fringes appear just after the cracks' formation, revealing delamination of the deposit from the substrate[14]. This delamination starts from the original contact line and progresses towards the central region of the drop. 

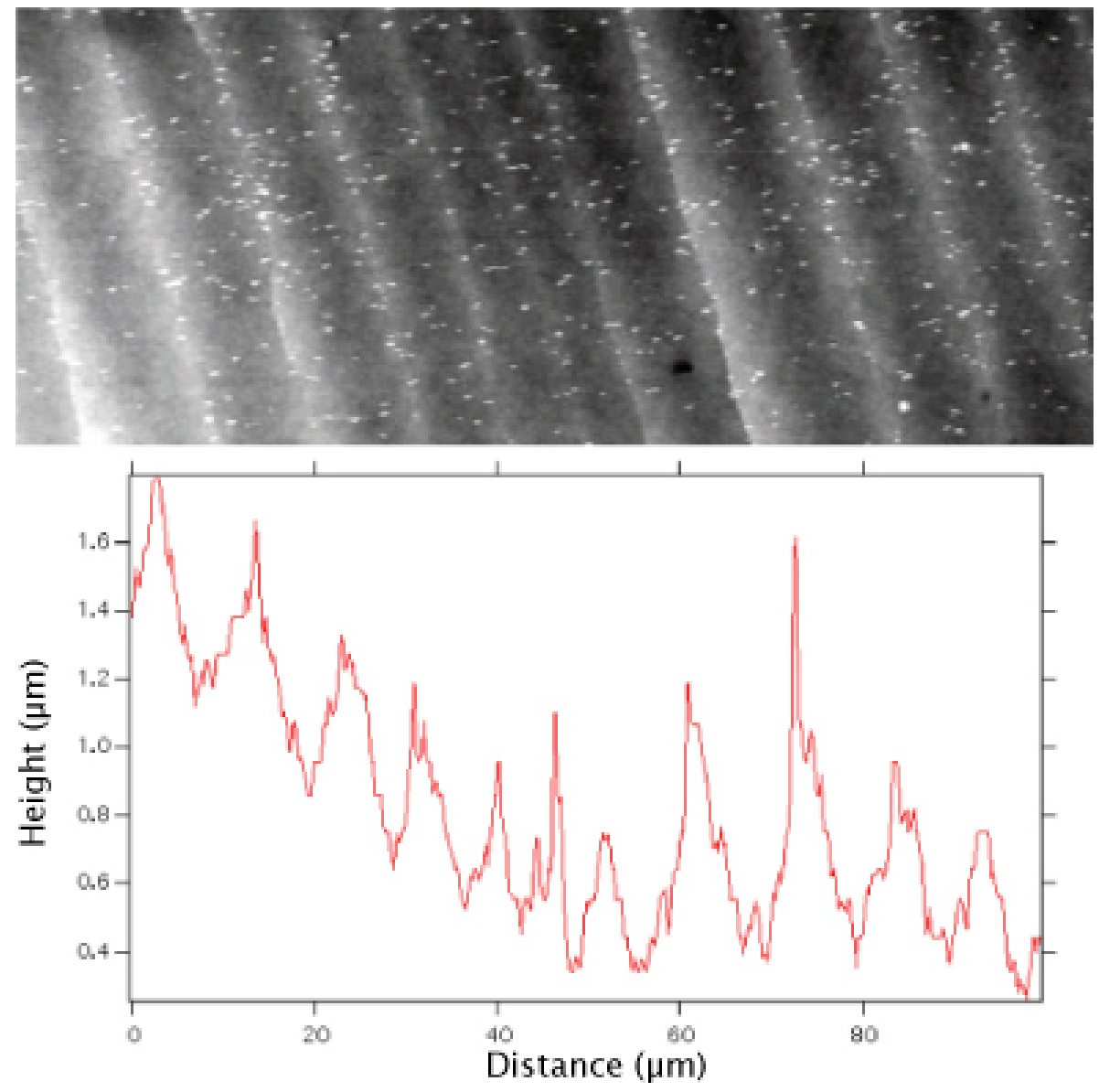

Figure 3: Pattern observation using AFM microscopy.

\section{Fluorescence microscopy experiments}

We were able to use fluorescence microscopy to harvest more information on the deposit structure. The experiment was performed using $50 \mathrm{~nm}$ fluorescent particles in a more dilute concentration (1\%). These particles cannot be tracked individually, but we can have a fairly good approximation of their number using the intensity of the fluorescent light received, since the thickness involved is of the order of $100 \mu \mathrm{m}$. Using this intensity, we were able to 


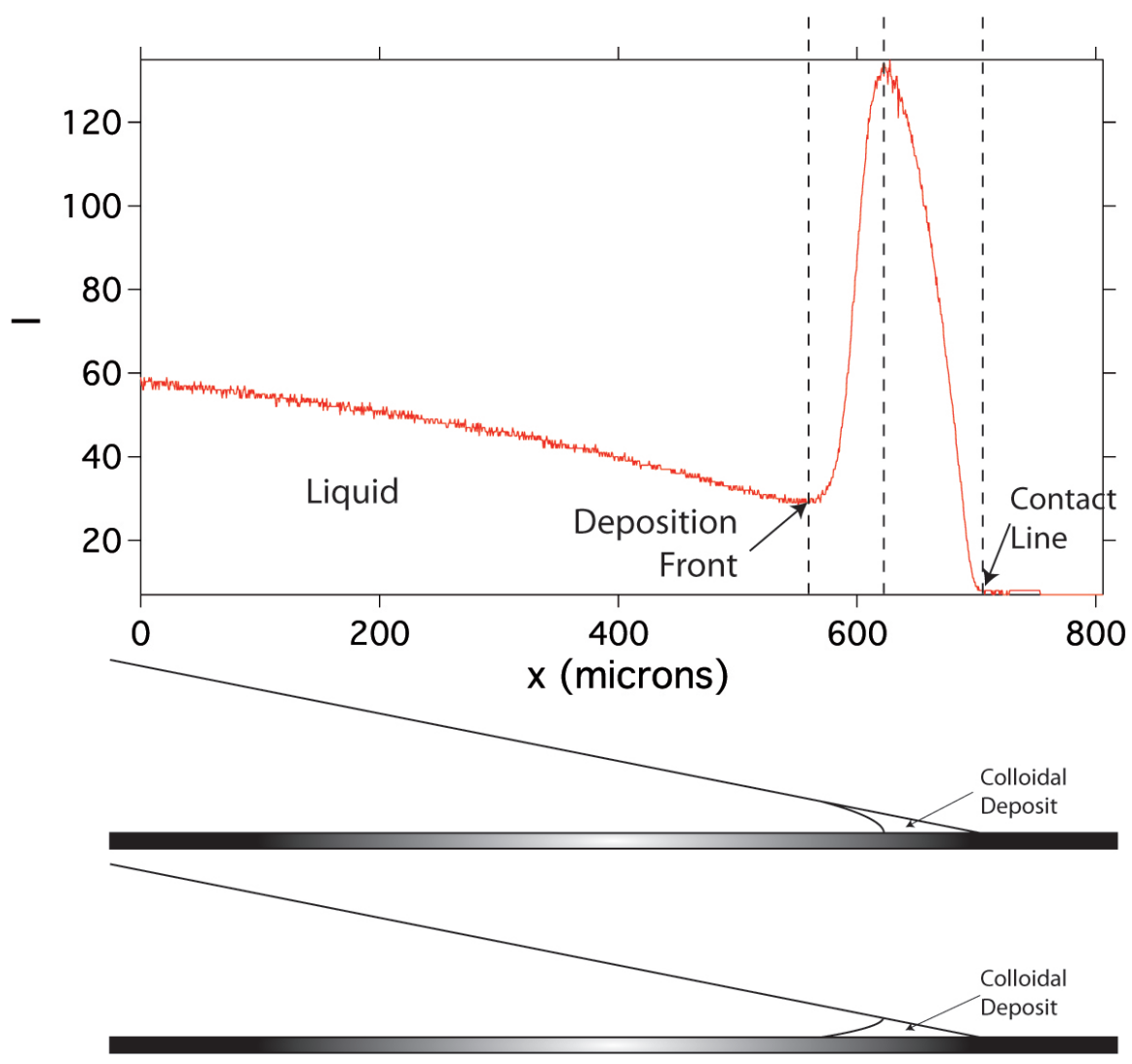

FiguRE 4: Typical shape of a profile extracted from a fluorescent microscopy experiment : fluorescent intensity $(I)$ in arbitrary units versus position $x$ in microns. The left part of the fluorescence peak can be explained in two ways, as described by the two schematics.

extract the profile of the deposit using MATLAB, as shown in Figures 4,5.

A typical profile is composed of two parts, as seen on 4 : one ascending part, close to the contact line, and one descending part, close to the deposition front. The descending part could correspond to two different structures as suggested by Figure 4, which cannot be discriminated using fluorescence microscopy : either the deposit grows along the glass substrate, or along the liquid-air interface, forming a colloidal skin.

In any case, these curves show us that there are two steps in the deposition 

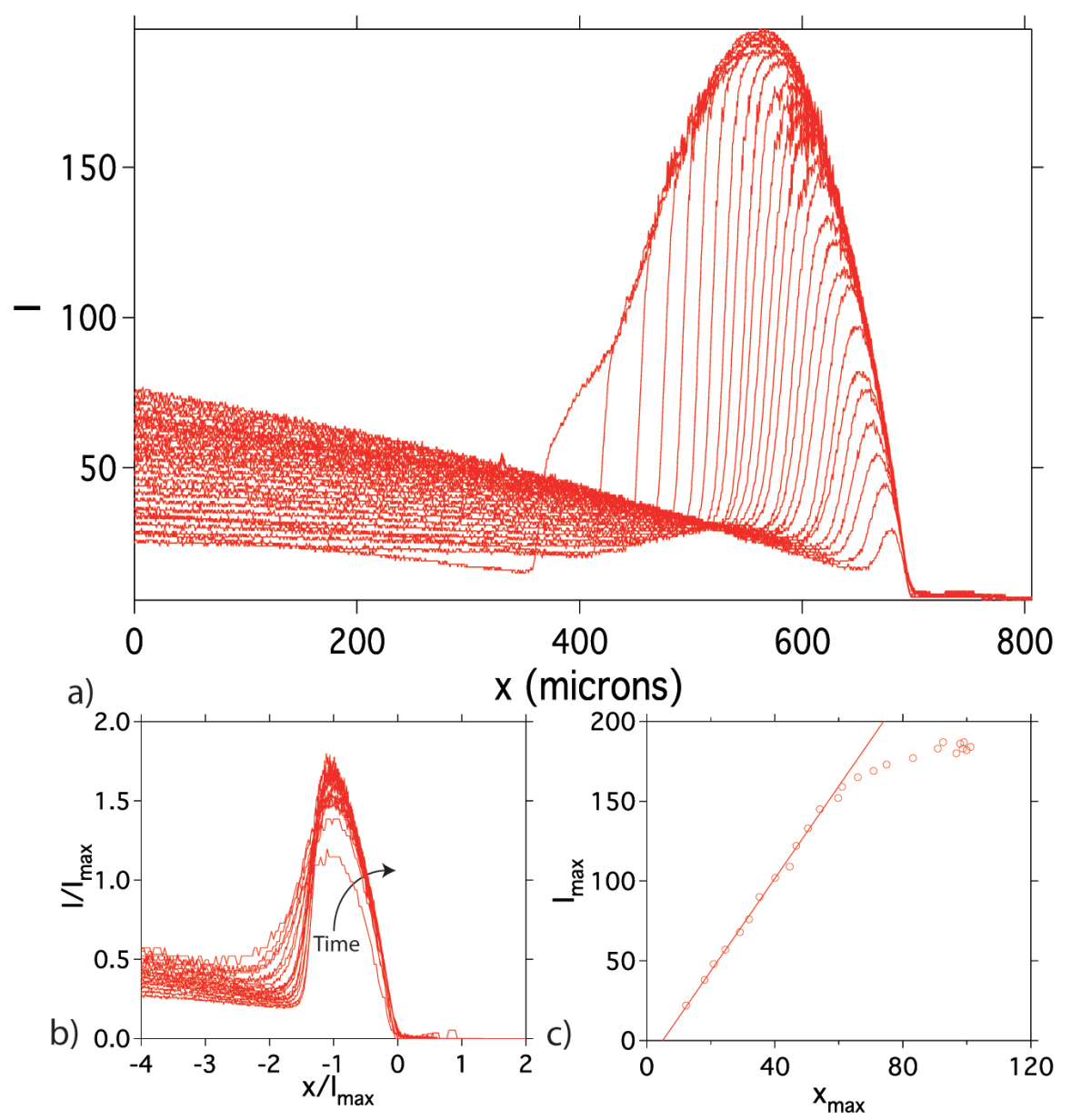

Figure 5: Shape of the deposit, using fluorescent particles (1\%concentration). a) Intensity profile obtained with $10 \times$ objective.Each curve is separated by 200s. b) Rescaling of profiles at early times using position of the maximum $\left(I_{\max }\right)$ for the fluorescence intensity $(I)$ and relative position $(x)$. c) Maximum fluorescence $\left(I_{\max }\right)$ intensity as a function of position of the maximum $\left(x_{\max }\right)$ : the early time shows a linear dependance, consistent with our growth scenario.

mechanism :

- at short times, the deposit grows as if one was building a wedge, with a nearly constant angle : both its height and spatial extent are increasing with time, keeping their ratio constant. This feature can be seen on 
5-b) : by rescaling the fluorescent profiles by dividing the fluorescence intensity by the maximal intensity observed and the position by the position of this maximal intensity, all profiles collapse together for the ascending part, with the exception of the first measurement. However,

5 -c) shows that the position $x_{\max }$ of the maximal intensity $I_{\max }$ confirms this behavior even at early times.

- at longer times, the growth seems different : the particles seem to be no longer in sufficient number to fill a wedge, and the maximal thickness of the deposit reaches at some time a maximum. Using the same rescaling as described above, we can see that the ascending part is no longer growing while the descending part is. This could be linked to a colloidal skin forming along the air-liquid interface, as shown in Figure 4. Close observation show motion of particles under the deposit, which agrees with this hypothesis. It would be interesting to further investigate how this descending profile matches the fadeout profile investigated recently by Witten[27].

As we shall see, while the second regime is quite complex, the first one can be described by a simple model : while the contact line is pinned to the substrate, particles are being driven by the hydrodynamic flow induced by evaporation to build a wedge-like deposit.

\section{Colloidal deposit growth : a simple model}

We have developed a model for the initial growth of a colloidal deposit while the contact line is pinned, whose complexity is intermediate between the qualitative method from Deegan [11] and the more rigorous one developed by Popov[12] and later Zheng[28]. It was inspired by an approach introduced 
(a)
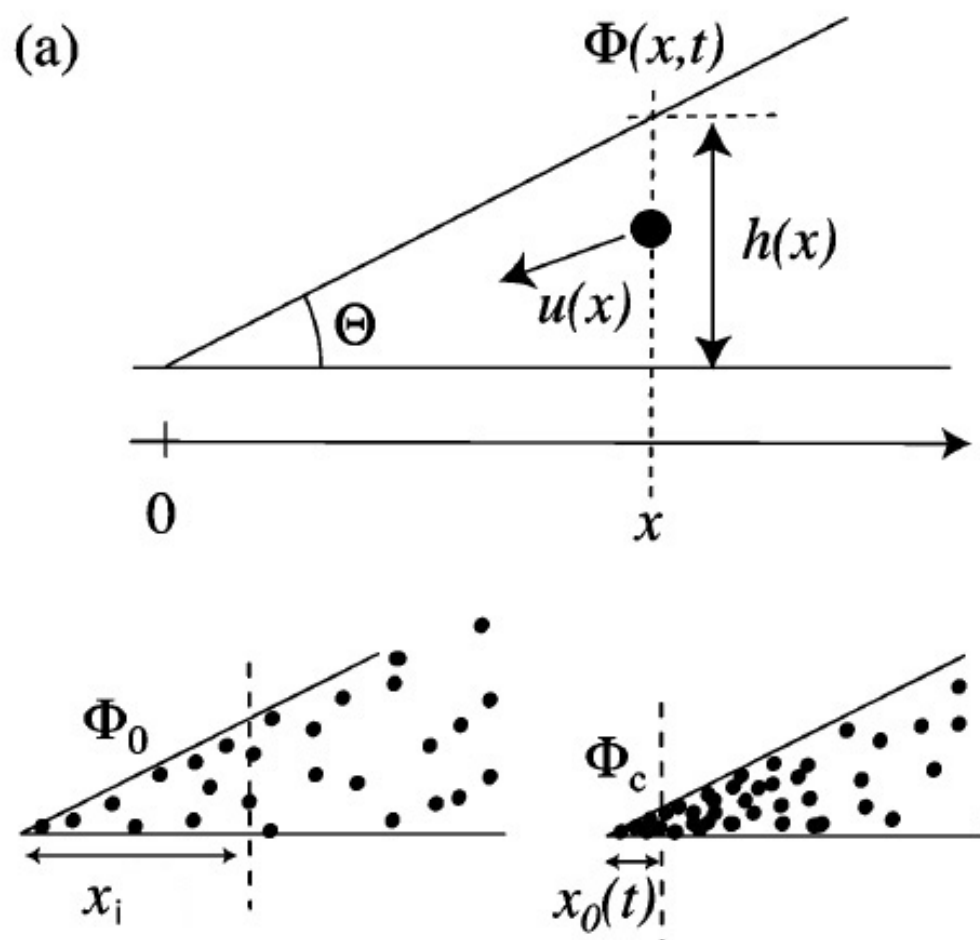

(b)

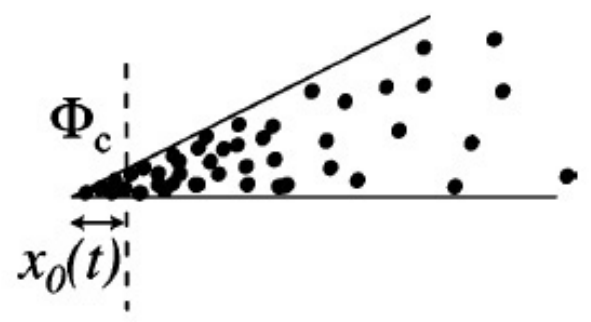

(c)

Figure 6: (a) Notation used to describe the motion of a particle inside the liquid wedge. (b) Initial concentration field at time $t=0$ : the concentration is homogeneous. (c) Situation at time $t$ : particles are concentrated at the contact line generating a solid defect of size $x_{0}$ inside which the concentration is equal to $\Phi_{c}$. At time $\mathrm{t}$, all of the particles inside the solid defect come from a distance smaller than $x_{i}$ from the contact line that is in fact proportional to $x_{0}$ (see eq. (1)). 
by Rio et al [13], in which we have corrected a mistake. We first reconsider this approach and correct it, and then show how to include more complex effects initially neglected (motion of the deposition front, change of contact angle with time...).

\subsection{Assumptions}

In [13], as well as here up to section 5-2, it is assumed that :

- the evaporation rate is of the form calculated by Deegan et al [8], $J(x)=J_{0} x^{-\frac{1}{2}}, J_{0}$ being given by $J_{0}=\frac{D_{g}}{\sqrt{\lambda}} \frac{c_{w}^{\text {sat }}}{\rho_{w}}$ where $D_{g}$ is the diffusion constant of evaporated solvent in air, $c_{w}^{\text {sat }}$ its mass concentration in air at saturation, $\lambda$ a typical length scale (of the order of the radius $2 R$ of the droplet) and $\rho_{w}$ its mass density. In our problem, using value for water evaporating in air, $D_{g}=24 * 10^{-6} \mathrm{~m}^{2} \mathrm{~s}^{-1}, c_{w}^{\text {sat }}=24 \mathrm{~g} \cdot \mathrm{m}^{-3}$, $\rho_{w}=1000 \mathrm{~kg} \cdot \mathrm{m}^{-3}, 2 R \approx 4 \mathrm{~mm}: J_{0}$ is of the order of $5 \times 10^{-10} \mathrm{~m}^{\frac{3}{2}} \cdot \mathrm{s}^{-1}$.

- the evaporation singularity is always located at the original contact line, and is not affected by the deposit.

- Marangoni flows are neglected and the droplets are of small contact angle.

\subsection{A first model}

We will call $x_{0}(t)$ the position of the deposition front, $x_{i}$ the position at $t=0$ of a particle for which $x(t)=x_{0}(t)$. Strictly speaking, this quantity depends on $t$ and measures the position of the "capture line" at $t$ : all the particles that satisfy $0<x<x_{i}$ at $t=0$ will be inside the solid deposit at $t$. Mass conservation can be written as :

$$
\Phi_{0} \theta x_{i}^{2}=\Phi_{c} \theta x_{0}(t)^{2}
$$


where $\theta$ is the contact angle, which in this regime is independent of time. $\Phi_{c}$ is the concentration of the silica particles inside the deposit (we will assume a close-packing concentration, $\left.\Phi_{c}=0.6\right)$, and $\Phi_{0}$ the initial concentration of particles in the liquid. Note that even though defined for $t=0, x_{i}$ is proportional to $x_{0}(t)$.

The motion of the liquid, using the set of hypothesis described earlier, is then supposed to be only dependent on evaporation. The velocity of a particle $U(x)=\frac{d x}{d t}$ can then be written as :

$$
\frac{d x}{d t}=\frac{J_{0}}{\theta} x^{-\frac{1}{2}}
$$

which leads to :

$$
x(t)=\left(-\frac{3}{2} J_{0} t+x(0)^{\frac{3}{2}}\right)^{\frac{2}{3}} \frac{1}{\theta^{\frac{2}{3}}}
$$

The solid deposit has a size $x_{0}(t)$, and the deposition front is formed by particles for which $x(t)=x_{0}(t)$. The particles inside the solid deposit originate from the domain $\left[0, x_{i}(t)\right]$ where :

$$
x_{i}(t)=x(0)=\left(\frac{3}{2} J_{0} t+x(t)^{\frac{3}{2}}\right)^{\frac{2}{3}} \frac{1}{\theta^{\frac{2}{3}}}
$$

Combined with (3) and the mass conservation equation (1), the deposit will grow as :

$$
x_{0}(t)=\left[\frac{3 J_{0} t}{\theta\left({\frac{\Phi_{c}}{\Phi_{0}}}^{\frac{3}{4}}-1\right)}\right]^{\frac{2}{3}}
$$

Note here that the $\left(J_{0} t\right)^{\frac{2}{3}}$ dependence can be guessed by a dimensional argument, while the prefactor differs from the one found in Rio et al[13] by a factor $\left(\frac{2}{\theta}\right)^{\frac{2}{3}}$. 
One can compare this result to the one given by Popov[12] in Eq. (47), in the case of $\phi_{0} \ll \phi_{c}$ which translates with our notations as :

$$
x_{0}(t)=\sqrt{\frac{\phi_{0}}{\phi_{c}}}\left(\frac{3 J_{0} t}{\theta}\right)^{\frac{2}{3}}
$$

In this dilute approximation, $\frac{\Phi_{c}}{\Phi_{0}} \gg 1$ and Eq. (5) gives the same result. Each of the two models has his own interest : the present model is simpler and provides a solution in principle for any value of $\phi_{0}$ (although perhaps only an estimate at large $\phi_{0}$ ), while the more complete approach developed by Popov and Zheng gives other information and is supposed to be valid for longer time scales. Note however that this one implies that the maximal height of the deposit and its maximal length $x_{0}$ are reached at the same time $t_{f}$, which does not fit our fluorescence profile at long times (see 4). Our description from the first principles is also unable to predict this fact.

\subsection{Introducing a moving singularity}

However, it is quite obvious that the liquid does not flow in the same way inside and outside of the deposit. Outside of the deposit, the liquid can flow without obstacle towards the surface where it evaporates, while in the deposit it has to permeate across the porous medium formed by the deposed particles. It seems thus rather natural to imagine that the evaporation of liquid will be very reduced at the surface of the deposit, and that on the contrary, it can diverge only in the liquid phase containing the particles not yet deposed. A rough simplification of this situation consists in assuming no evaporation at the surface of the deposit and a "free" evaporation at the liquid surface, which is equivalent to say that there is an effective contact line at the deposition front where evaporation diverges, this effective contact 
line following exactly the motion of the deposition front. At least, we can expect that the reality should be somewhere between the two limit cases : static and moving singularity. In the moving singularity approximation, the evaporation profile is given by :

$$
J(x, t)=J_{0}\left(x-x_{0}(t)\right)^{-\frac{1}{2}}
$$

The particles velocity $U(x)=\frac{d x_{p}}{d t}$ will follow the equation :

$$
U(x)=\frac{1}{h} \int_{0}^{x} \frac{J_{0}}{\sqrt{x^{\prime}}} d x^{\prime}
$$

which, assuming $h \approx \theta x$ leads to :

$$
2 J_{0} \sqrt{x-x_{0}(t)}=\theta x U(x)
$$

We can change the unknown quantity in (9) by introducing $\xi(t)=x-x_{0}(t)$, and this equation will become :

$$
\frac{d x}{d t}=-\frac{2 J_{0}}{\theta} \frac{\sqrt{\xi}}{\xi+x_{0}}
$$

Let us now assume that the deposit is growing very slowly, compared with the velocity of the liquid, which translates as $\frac{d x_{0}}{d t} \ll \frac{d x}{d t}$. We have checked this assumption by introducing bigger particles inside the colloidal suspension, such as $3 \mu \mathrm{m}$ particles, which can be seen individually using magnification : their velocity is of the order of $1 \mathrm{~mm} . \mathrm{s}^{-1}$, whereas the deposit grows at the speed of about $1 \mu m . s^{-1}$. We can then use this assumption in 10 :

$$
\frac{\xi+x_{0}}{\sqrt{\xi}} d \xi=-\frac{2 J_{0}}{\theta} d t
$$

We now have to integrate (11) between $t=0$ and $t$, for a particle starting from $x_{i}$ and arriving at $x_{0}$ : 


$$
\begin{aligned}
& -\xi(0)=x_{i}-x_{0}(t)=\left(\sqrt{\frac{\Phi_{c}}{\Phi_{0}}}-1\right) x_{0}(t) \\
& -\xi(t)=x_{0}(t)-x_{0}(t)=0
\end{aligned}
$$

Integration yields :

$$
x_{0}(t)=\left(\frac{J_{0}}{\theta\left[\frac{1}{3}\left(\sqrt{\frac{\Phi_{c}}{\Phi_{0}}}-1\right)^{\frac{3}{2}}+\left(\sqrt{\frac{\Phi_{c}}{\Phi_{0}}}-1\right)^{\frac{1}{2}}\right]}\right)^{\frac{2}{3}} t^{\frac{2}{3}}
$$

We can notice that the power law is the same as the one found by Rio et $a l$, which comes as no surprise given that the $\left(J_{0} t\right)^{\frac{2}{3}}$ can be guessed using dimensional arguments.

The ratio between the deposit predicted with a moving singularity and the one predicted with a fixed singularity is then :

$$
r=\left(\frac{\frac{\Phi_{c} \frac{3}{4}}{\Phi_{0}}-1}{\left[\frac{1}{3}\left(\sqrt{\frac{\Phi_{c}}{\Phi_{0}}}-1\right)^{\frac{3}{2}}+\left(\sqrt{\frac{\Phi_{c}}{\Phi_{0}}}-1\right)^{\frac{1}{2}}\right]}\right)^{\frac{2}{3}}
$$

which is independent of the contact angle $\theta$ and decreases with the concentration of particles $\Phi_{0}$, but does not depend on the evaporation rate $J_{0}$. This ratio, along with the two different prefactors given by the two different models, are plotted against particle concentration on Figure7.In our experiments, $r \approx 0.92$ which shows that, at least theoretically, the effect of a moving singularity is not negligible.

\subsection{Variable contact angle model}

In principle, to apply this model to a droplet, one has to consider that the amount of liquid is limited by the volume of the droplet, and this volume is constantly decreasing due to evaporation which implies some change of 


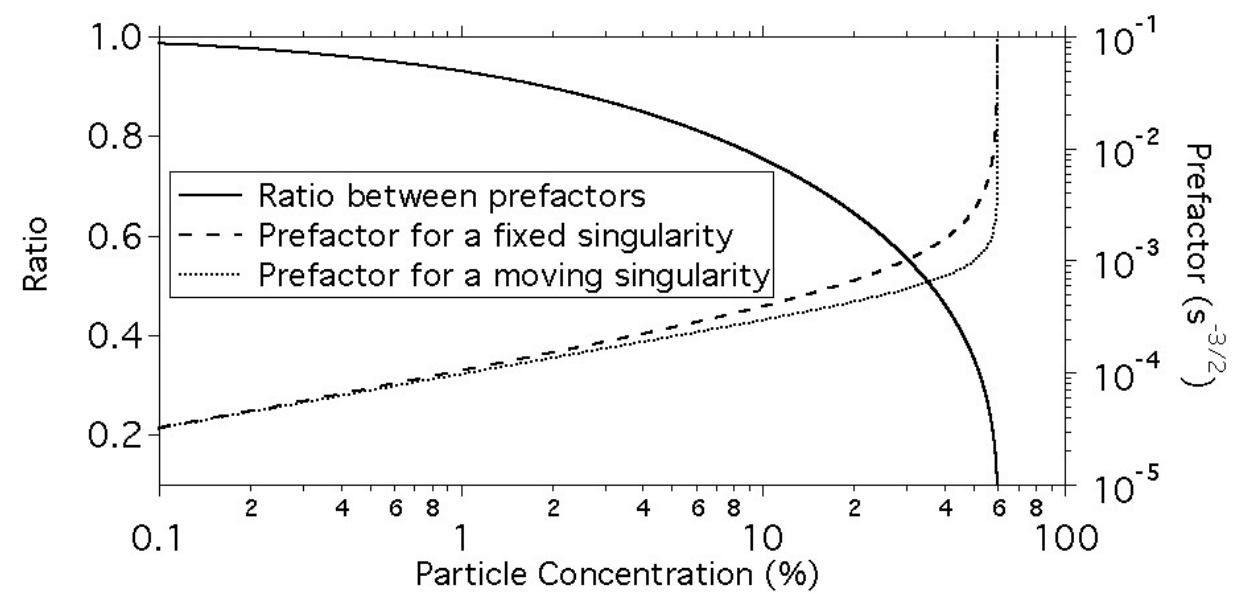

Figure 7: Prefactors given by the fixed singularity model (dashed line) and by the moving singularity (dotted line). The solid line gives the ratio between the two prefactors. The deposit concentration is here fixed at $\phi_{c}=60 \%$.

contact angle with time, not discussed above. Deegan et al[8] showed that the loss of volume is proportional to the radius of the droplet :

$$
V=V_{0}-2 \pi R \int_{0}^{R} J(x)=V_{O}-2 \pi R^{\frac{3}{2}} J_{0}
$$

Where $V_{0}$ is the initial volume of the droplet.

If the radius is smaller than the capillary length, the shape of the droplet will be a spherical cap. If the contact angle $\theta$ is small, we then have :

$$
V=\frac{\pi}{4} R^{3} \theta(t)
$$

which leads to :

$$
\begin{aligned}
& \theta(t)=\left(V_{O}-2 \pi R^{\frac{3}{2}} J_{0} t\right) \frac{4}{\pi R^{3}} \\
& \theta(t)=\theta_{0}-8 R^{-\frac{3}{2}} J_{0} t
\end{aligned}
$$

This will change (11) into :

$$
\frac{\xi+x_{0}}{\sqrt{\xi}} d \xi=-\frac{2 J_{0}}{\theta_{0}-8 R^{-\frac{3}{2}} J_{0} t} d t
$$


Which integrates as :

$$
x_{0}(t)=-\Psi \ln \left(\theta_{0}-8 R^{-\frac{3}{2}} J_{0} t\right)^{\frac{2}{3}}
$$

where

$$
\Psi=\left(\frac{1}{8 R^{-\frac{3}{2}}\left(\frac{1}{3}\left(\sqrt{\frac{\Phi_{c}}{\Phi_{0}}}-1\right)^{\frac{3}{2}}+\left(\sqrt{\frac{\Phi_{c}}{\Phi_{0}}}-1\right)^{\frac{1}{2}}\right)}\right)^{\frac{2}{3}}
$$

At short times, the contact angle is close to the initial contact angle $\theta_{0}$, enabling us the approximation $\ln \left(\theta_{0}-8 R^{-\frac{3}{2}} J_{0} t\right) \approx-8 \frac{R^{-\frac{3}{2}}}{\theta_{0}} J_{0} t-\frac{\left(8 \frac{R^{-\frac{3}{2}}}{\theta_{0}} J_{0} t\right)^{2}}{2}$. The first order gives us the same expressions as expression (12), and a correction scaling as $t^{\frac{4}{3}}$. This effect is negligible in the experiments discussed here.

\subsection{Experimental verification}

To test our theory, we have measured the spatial extent $x_{0}$ of the deposit from our visualizations of its growth. Simply laying a sessile colloidal suspension droplet on a glass substrate, we observe it with a microscope. Size $x_{0}$ of the deposit is subsequently given by measuring the distance between the contact line and the deposition line through a imaging software (NIS Elements, Nikon), as indicated on 2. We can then plot these measurements as in 8. Our model predicts a linear relationship between $x_{0}^{\frac{3}{2}}$ and $t$, which is clearly shown for short times.

As mentioned in section 2, we have observed through a sideview visualization that for our conditions, colloidal drops deposited on untreated fresh glass slides exhibit a contact angle of approximately $35^{\circ}$, measured using 


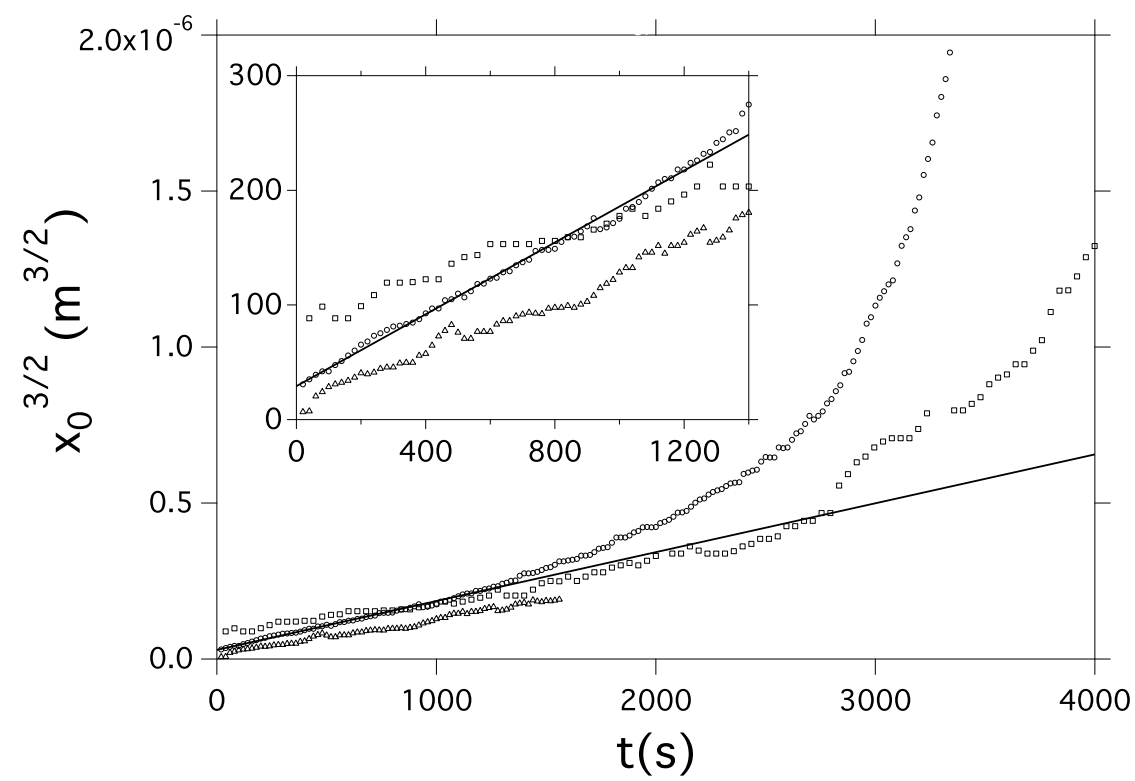

Figure 8: Growth of the deposit length $x_{0}$ vs time $t$. The quantity $x_{0}^{3 / 2}$ has been plotted on the vertical axis, so that linear dependence on $t$ is expected at short times. The three traces represent three different experiments performed on different days with different temperature and humidity. Inset shows detail at early times. The solid line is a fit to a power law $t^{\frac{3}{2}}$

Drop Shape Analyser 100 by Krüss, which is intermediate between plasmacleaned glass (total wetting condition) and old glass previously exposed to ambient air. The value of $J_{0}$ can be extrapolated from a fit, as the one seen in 8 . The result is $J_{0} \approx 4.5 \times 10^{-10} \mathrm{~m}^{\frac{3}{2}} \mathrm{~S}^{-1}$ with the first model, and $J_{0} \approx 7.2 \times 10^{-10} \mathrm{~m}^{\frac{3}{2}} \mathrm{~S}^{-1}$ with the moving singularity model, while estimation of the evaporation give a range of $J_{0} \approx 5$ to $10 \times 10^{-10} \mathrm{~m}^{\frac{3}{2}} \mathrm{~S}^{-1}$. Those orders of magnitude are satisfactory, but quantitative monitoring of the evaporation during the experiment would be needed to discriminate between the two versions of our model. In any case, both are based on the same physical ideas that seem to hold here with a good accuracy. 


\section{Conclusion and Acknowledgements}

To summarize, we have observed for the first time the growth of the "coffee stain" microscopically, in real-time conditions. This allowed us to understand the different steps of such growth, from the first growth inside a wedge to the delamination process. Moreover patterns were observed inside this deposit, related to a height modulation of the surface. We were also able to work out a model improving the prediction of the growth of the deposit in the first stage by introducing a moving singularity. As this model is strongly dependent on the form chosen for the evaporation field, one can see it and its experimental verification as further evidence in favor of the theory provided by Deegan et al [8]. However, the difference observed experimentally between the two versions of our models is based on the estimate of the evaporation, and better measurement would be appropriate to better discriminate between them.

Further studies are necessary to understand the patterns observed, especially the selection mechanism of the stripes, as well as to discriminate between the deposit scenarii leading to the possible existence of a colloidal skin. One may also want to use such a deposition method in order to fabricate nanostructured materials [29]. Finally, an additional model would be needed for the understanding of the second step of the deposit growth, when the colloidal particles are highly diluted.

After completion of this paper, we became aware of another numerical study that gives results very similar to ours with respect to the deposit profile[30].

We would like to acknowledge the French Delégation Générale de l'Armement (D.G.A.), Agence Nationale de la Recherche (A.N.R.-DEPSEC pro- 
gram) and National Science Foundation (grant : CBET-0730251) for funding. We also thank A.Z. France for supply of colloidal suspension and Chi-Tuong Pham and Tom Witten for fruitful discussions.

\section{Références}

[1] Maenosono, S.; Dushkin, C.; Saita, S. ; Yamaguchi, Y. Langmuir 1999, $15,957$.

[2] Belleville, P. Revue verre 2000, 6 .

[3] Qu, D. ; Ramé, E. ; Garoff, S. Phys. Fluids 2002, 14, 1154.

[4] Prevo, B.; Velev, O. Langmuir 2004, 20, 2099-2107.

[5] Berteloot, G.; Pham, C.-T.; Daerr, A.; Lequeux, F. EuroPhys. Lett. 2008, 83, 14003.

[6] Koh, Y.; Wong, C. Langmuir 2006, 22, 897-900.

[7] Adachi, E.; Dimitrov, A. S.; Nagayama, K. Langmuir 1995, 11, 1057.

[8] Deegan, R.; Bakajin, O. ; Dupont, T.; Huber, G.; Nagel, S.; Witten, T. Nature 1997, 389, 827-828.

[9] Jung, Y.; Kajiya, T.; Yamaue, T.; Doi, M. Jap. Journal of App. Physics. 2009, 48 .

[10] Liu, X.; Guan, Y.; Ma, Z.; Liu, H. Langmuir 2004, 20, 10278-10282.

[11] Deegan, R. D.; Bakajin, O.; Dupont, T.; ans S. R. Nagel, G. H. ; Witten, T. Phys. Rev. E 2000, 62, 756-765. 
[12] Popov, Y. Phys. Rev. E 2005, 71, 036613.

[13] Rio, E. ; Daerr, A.; Lequeux, F. ; Limat, L. Langmuir 2006, 22, 3186.

[14] Pauchard, L. Europhys. Lett. 2006, 74, 188.

[15] Man, W.; Russel, W. Phys. Rev. Lett. 2008, 100.

[16] Ristenpart, W. D.; Kim, P. G. ; Domingues, C. ; Wan, J. ; Stone, H. A. Phys. Rev. Lett. 2007, 99, 234502.

[17] Xu, X.; Luo, J. Appl. Phys. Lett. 2007, 91, 124102.

[18] Hu, H.; Larson, R. J. Phys.Chem. B 2002, 106, 1334-1344.

[19] Bhardwaj, R.; Fang, X.; Attinger, D. New Journal of Physics 2009, 11.

[20] Hu, H. ; Larson, R. G. Langmuir 2005, 21, 3972-3980.

[21] Kajiya, T.; Monteux, C.; Narita, T.; Lequeux, F. ; Doi, M. Langmuir 2009, 25, 6934-6939.

[22] Flesselles, J.-M. ; Simon, A. J. ; Liebchaber, A. Advance in Physics 1991, $40,1-51$.

[23] Schroers, J.; Johnson, W. L. Phys. Rev. Lett. 2004, 93.

[24] Pauchard, L.; Parisse, F.; Allain, C. Phys. Rev. E 1999, 59, 3737-3740.

[25] Annarelli, C.; Fornazero, J.; Bert, J.; Colombani, J. Eur. Phys. J. E 2001, 5, 599-603. 
[26] Sommer, A.; Rozlosnik, N. Crys. Growth 6 Design 2005, 5, 551-557.

[27] Witten, T. Eur. Phys. Letters 2009, 86.

[28] Zheng, R. Eur. Phys. Journal E 2009, 29, 205-218.

[29] Nagayama, K. Colloids and Surfaces A 1996, 109, 363-374.

[30] Bhardwaj, R.; Fang, X.; Somasundaran, P.; Attinger, D. Langmuir 2010, 26, 7833-7842. 